\title{
On the exact solutions of a modified Kortweg de Vries type equation and higher-order modified Boussinesq equation with damping term
}

\section{Dimpho Millicent Mothibi and Chaudry Masood Khalique*}

\section{"Correspondence:}

Masood.Khalique@nwu.ac.za International Institute for Symmetry

Analysis and Mathematical Modelling, Department of

Mathematical Sciences, North-West

University, Mafikeng Campus, Private Bag X 2046, Mmabatho,

2735, Republic of South Africa

\begin{abstract}
In this paper, we obtain exact solutions of two nonlinear evolution equations, namely the modified Kortweg de Vries equation and the higher-order modified Boussinesq equation with damping term. The method employed to obtain the exact solutions is the $\left(G^{\prime} / G\right)$-expansion method. Traveling wave solutions of three types are obtained and these are the solitary waves, periodic and rational.
\end{abstract}

Keywords: modified Kortweg de Vries equation; modified Boussinesq equation; $\left(G^{\prime} / G\right)$-expansion method; traveling wave solutions

\section{Introduction}

In this paper, we consider two nonlinear evolution equations, namely the modified Kortweg de Vries equation [1]

$$
u u_{x x t}-u_{x} u_{x t}-4 u^{3} u_{t}+4 u u_{x x x}-4 u_{x} u_{x x}-16 u^{3} u_{x}=0
$$

and the higher-order modified Boussinesq equation with damping term [2]

$$
u_{t t}+\alpha u_{t x x}+\beta u_{x x x x}+\gamma\left[6 u\left(u_{x}\right)^{2}+3 u^{2} u_{x x}\right]=0 .
$$

It is well known that nonlinear evolution equations, such as (1) and (2), are widely used as models to describe physical phenomena in different fields of applied sciences such as plasma waves, solid state physics, plasma physics and fluid mechanics. One of the basic physical problems for these models is to obtain their exact solutions for the better understanding of nonlinear models [1-22]. In the last few decades, a variety of effective methods for finding exact solutions, such as the homogeneous balance method [3], the ansatz method $[4,5]$, the variable separation approach [6], the inverse scattering transform method [7], the Bäcklund transformation [8], the Darboux transformation [9], Hirota's bilinear method [10], the reduction mKdV equation method [11], the tri-function method $[12,13]$, the projective Riccati equation method [14], the sine-cosine method [15], the Jacobi elliptic function expansion method [16, 17], the $F$-expansion method [18] and the

(c) 2013 Mothibi and Khalique; licensee Springer. This is an Open Access article distributed under the terms of the Creative Commons Attribution License (http://creativecommons.org/licenses/by/2.0), which permits unrestricted use, distribution, and reproduction in any medium, provided the original work is properly cited. 
exp-function expansion method [19] and many others, were successfully applied to nonlinear differential equations.

Although a great deal of research work has been devoted to finding different methods to solve nonlinear evolution equations, there is no unique method. In 2007 Wang et al. [20] proposed a new method referred to as the $\left(G^{\prime} / G\right)$-expansion method for finding traveling wave solutions of nonlinear evolution equations. This paper showed that the $\left(G^{\prime} / G\right)$ expansion method is an effective method for finding exact solutions of nonlinear evolution equations. It has been extensively used by various researchers (see, for example, papers $[20-22])$ in a variety of scientific fields. The key ideas of the method are that the traveling wave solutions of a complicated nonlinear evolution equation can be constructed by means of various solutions of a second-order linear ordinary differential equation [20].

In this work, our main focus is on equations (1) and (2). We derive the traveling wave solutions of the two equations by using the $\left(G^{\prime} / G\right)$-expansion method. The paper is organized as follows. In Section 2, we describe the $\left(G^{\prime} / G\right)$-expansion method. Exact solutions of the modified Kortweg de Vries equation (1) and the higher-order modified Boussinesq equation with damping term (2) are constructed in Section 3 using the $\left(G^{\prime} / G\right)$-expansion method. In Section 4, conclusion is given.

\section{Analysis of the $\left(G^{\prime} / G\right)$-expansion method}

The $\left(G^{\prime} / G\right)$-expansion method for finding exact solutions of nonlinear differential equations was introduced in [20]. Several researchers have recently applied this method to various nonlinear differential equations. They have shown that this method provides a very effective and powerful mathematical tool for solving nonlinear equations in various fields of applied sciences (see, for example, papers [20-22]).

Consider a nonlinear partial differential equation (NPDE), say, in two independent variables $x$ and $t$, given by

$$
P\left(u, u_{x}, u_{t}, u_{t t}, u_{x t}, u_{x x}, \ldots\right)=0 \text {, }
$$

where $u(x, t)$ is an unknown function, $P$ is a polynomial in $u$ and its various partial derivatives, in which the highest-order derivatives and nonlinear terms are involved. The essence of the $\left(G^{\prime} / G\right)$-expansion method is given in the following steps.

- Step 1 . The transformation $u(x, t)=U(z), z=x-v t$ reduces equation (3) to the ordinary differential equation (ODE)

$$
P\left(U,-v U^{\prime}, U^{\prime}, v^{2} U^{\prime \prime},-v U^{\prime \prime}, U^{\prime \prime}, \ldots\right)=0 .
$$

- Step 2. According to the $\left(G^{\prime} / G\right)$-expansion method, it is assumed that the traveling wave solution of equation (4) can be expressed by a polynomial in $\left(G^{\prime} / G\right)$ as follows:

$$
U(z)=\sum_{i=0}^{m} \alpha_{i}\left(\frac{G^{\prime}}{G}\right)^{i}
$$

where $G=G(z)$ satisfies the second-order linear ODE in the form

$$
G^{\prime \prime}+\lambda G^{\prime}+\mu G=0
$$


with $\alpha_{i}, i=0,1,2, \ldots, m, \lambda$ and $\mu$ being constants to be determined. The positive integer $m$ is determined by considering the homogenous balance between the highest-order derivatives and nonlinear terms appearing in ODE (4).

- Step 3. By substituting (5) into (4) and using the second-order ODE (6), collecting all terms with same order of $\left(G^{\prime} / G\right)$ together, the left-hand side of (4) is converted into another polynomial in $\left(G^{\prime} / G\right)$. Equating each coefficient of this polynomial to zero, yields a set of algebraic equations for $\alpha_{0}, \ldots, \alpha_{m}, \nu, \lambda, \mu$.

- Step 4. Assuming that the constants can be obtained by solving the algebraic equations in Step 3, since the general solution of (6) is known, then substituting the constants and the general solutions of (6) into (5) we obtain traveling wave solutions of the NPDE (3).

\section{Exact solutions of (1) and (2)}

In this section we construct traveling wave solutions of $\mathrm{mKdV}$ and modified Boussinesq equations by employing the $\left(G^{\prime} / G\right)$-expansion method.

\subsection{The modified Kortweg de Vries equation}

The modified KdV equation is given by [1]

$$
u u_{x x t}-u_{x} u_{x t}-4 u^{3} u_{t}+4 u u_{x x x}-4 u_{x} u_{x x}-16 u^{3} u_{x}=0
$$

where $u$ is a real-valued scalar function, $t$ is time and $x$ is a spatial variable.

As the first step, we transform the modified KdV type equation (7) to a nonlinear ordinary differential equation (ODE) using the traveling wave variable

$$
u(t, x)=F(z), \quad z=x-v t .
$$

Applying the above transformation, equation (7) transforms to the nonlinear ODE

$$
-v F F^{\prime \prime \prime}+v F^{\prime} F^{\prime \prime \prime}+4 v F^{3} F^{\prime}+4 F F^{\prime \prime \prime}-4 F^{\prime} F^{\prime \prime}-16 F^{3} F^{\prime}=0,
$$

which reduces to

$$
(4-v)\left[F F^{\prime \prime \prime}-F^{\prime} F^{\prime \prime}-4 F^{3} F^{\prime}\right]=0 .
$$

Hence if $v \neq 4$, we obtain

$$
F F^{\prime \prime \prime}-F^{\prime} F^{\prime \prime}-4 F^{3} F^{\prime}=0,
$$

where the prime denotes the derivative with respect to $z$.

The $\left(G^{\prime} / G\right)$-expansion method assumes the solution of equation (11) to be of the form

$$
F(z)=\sum_{i=0}^{M} \mathcal{A}_{i}\left(G^{\prime} / G\right)^{i}
$$

where $G(z)$ satisfies the second-order linear ODE with constant coefficients, viz.,

$$
G^{\prime \prime}+\lambda G^{\prime}+\mu G=0
$$

where $\lambda$ and $\mu$ are constants. 
The balancing procedure yields $M=1$, so the solution of the ODE (11) is of the form

$$
F(z)=\mathcal{A}_{0}+\mathcal{A}_{1}\left(G^{\prime} / G\right)
$$

Substituting (14) into (11), making use of the ODE (13), collecting all terms with same powers of $\left(G^{\prime} / G\right)$ and equating each coefficient to zero yield the following system of algebraic equations:

$$
\begin{aligned}
& \left(G^{\prime} / G\right)^{0}:-\mathcal{A}_{0} \mathcal{A}_{1} \lambda^{2} \mu-2 \mathcal{A}_{0} \mathcal{A}_{1} \mu^{2}+\mathcal{A}_{1}^{2} \lambda \mu^{2}+4 \alpha \mathcal{A}_{0}^{3} \alpha_{1} \mu=0 \\
& \left(G^{\prime} / G\right): 4 \mathcal{A}_{0}^{3} \mathcal{A}_{1} \lambda+12 \mathcal{A}_{0}^{2} \mathcal{A}_{1}^{2} \mu+\mathcal{A}_{1}^{2} \lambda^{2} \mu-\mathcal{A}_{0} \mathcal{A}_{1} \lambda^{3}-8 \mathcal{A}_{0} \mathcal{A}_{1} \lambda \mu=0 \\
& \left(G^{\prime} / G\right)^{2}:-7 \mathcal{A}_{1}^{2} \lambda^{2}-8 \mathcal{A}_{1}^{2} \mu-7 \mathcal{A}_{0} \mathcal{A}_{1} \lambda^{2}-8 \mathcal{A}_{0} \mathcal{A}_{1} \mu-2 \mathcal{A}_{1}^{2} \lambda \mu \\
& \quad+12 \mathcal{A}_{0} \mathcal{A}_{1}^{3} \mu+12 \mathcal{A}_{0}^{2} \mathcal{A}_{1}^{2} \lambda+4 \mathcal{A}_{0}^{3} \mathcal{A}_{1}=0 \\
& \left(G^{\prime} / G\right)^{3}: 4 \mathcal{A}_{1}^{4} \mu+12 \mathcal{A}_{0} \mathcal{A}_{1}^{3} \lambda+12 \mathcal{A}_{0}^{2} \mathcal{A}_{1}^{2}+4 \mathcal{A}_{1}^{2} \lambda^{2}+4 \mathcal{A}_{1}^{2} \mu \\
& \quad-12 \mathcal{A}_{0} \mathcal{A}_{1} \lambda=0 \\
& \left(G^{\prime} / G\right)^{4}: 4 \mathcal{A}_{1}^{4} \lambda+12 \mathcal{A}_{0} \mathcal{A}_{1}^{3}-7 \mathcal{A}_{1}^{2} \lambda-6 \mathcal{A}_{0} \mathcal{A}_{1}=0 \\
& \left(G^{\prime} / G\right)^{5}: 4 \mathcal{A}_{1}^{4}-4 \mathcal{A}_{1}^{2}=0
\end{aligned}
$$

Solving this system of algebraic equations, with the aid of Mathematica, we obtain

$$
\mathcal{A}_{0}=\frac{\lambda}{2}, \quad \mathcal{A}_{1}=1
$$

Substituting these values of $\mathcal{A}_{0}, \mathcal{A}_{1}$ and the corresponding solution of ODE (13) into (14), we obtain three types of traveling wave solutions of equation (7). These are as follows.

Case 1: When $\lambda^{2}-4 \mu>0$, we obtain the hyperbolic function solutions

$$
u_{1}(t, x)=\mathcal{A}_{0}+\mathcal{A}_{1}\left(-\frac{\lambda}{2}+\delta_{1} \frac{C_{1} \sinh \left(\delta_{1} z\right)+C_{2} \cosh \left(\delta_{1} z\right)}{C_{1} \cosh \left(\delta_{1} z\right)+C_{2} \sinh \left(\delta_{1} z\right)}\right)
$$

where $z=x-v t, \delta_{1}=\frac{1}{2} \sqrt{\lambda^{2}-4 \mu}, C_{1}$ and $C_{2}$ are arbitrary constants.

Case 2: When $\lambda^{2}-4 \mu<0$, we obtain the trigonometric function solutions

$$
u_{2}(t, x)=\mathcal{A}_{0}+\mathcal{A}_{1}\left(-\frac{\lambda}{2}+\delta_{2} \frac{-C_{1} \sin \left(\delta_{2} z\right)+C_{2} \cos \left(\delta_{2} z\right)}{C_{1} \cos \left(\delta_{2} z\right)+C_{2} \sin \left(\delta_{2} z\right)}\right),
$$

where $z=x-v t, \delta_{2}=\frac{1}{2} \sqrt{4 \mu-\lambda^{2}}, C_{1}$ and $C_{2}$ are arbitrary constants.

Case 3: When $\lambda^{2}-4 \mu=0$, we obtain the rational function solutions

$$
u_{3}(t, x)=\mathcal{A}_{0}+\mathcal{A}_{1}\left(-\frac{\lambda}{2}+\frac{C_{2}}{C_{1}+C_{2} z}\right)
$$

where $z=x-v t, C_{1}$ and $C_{2}$ are arbitrary constants. 


\subsection{Higher-order modified Boussinesq equation with damping term}

We now consider the modified Boussinesq equation with damping term [2] given by

$$
u_{t t}+\alpha u_{t x x}+\beta u_{x x x x}+\gamma\left[6 u\left(u_{x}\right)^{2}+3 u^{2} u_{x x}\right]=0
$$

where $u$ is a real-valued scalar function, $t$ is time, $x$ is a spatial variable and $\alpha, \beta, \gamma$ are nonzero real constants.

Following the same procedure of the previous subsection equation (25) is transformed to the following ODE:

$$
v^{2} U^{\prime \prime}-\alpha \nu U^{\prime \prime \prime}+\beta U^{\prime \prime \prime \prime}+\gamma\left[6 U\left(U^{\prime}\right)^{2}+3 U^{2} U^{\prime \prime}\right]=0,
$$

where the prime denotes the derivative with respect to $z$. Balancing the order of $U^{\prime \prime \prime \prime}$ and $U^{2} U^{\prime \prime}$ in (26) yields $M=1$. The solution to equation (26) is also assumed to be of the form

$$
U(z)=a_{0}+a_{1}\left(G^{\prime} / G\right) .
$$

Substituting (27) into (26) and making use of (13), we obtain the following algebraic system of equations in terms of $a_{0}, a_{1}$, by equating all coefficients of the functions $\left(G^{\prime} / G\right)^{i}$ to zero.

$$
\begin{aligned}
& \left(G^{\prime} / G\right)^{0}: a_{1} \mu \lambda v^{2}+2 a_{1} \mu^{2} \alpha \nu+a_{1} \mu \lambda^{2} \alpha \nu+8 a_{1} \mu^{2} \lambda \beta+a_{1} \mu \lambda^{3} \beta \\
& +6 a_{0} a_{1}^{2} \mu^{2} \gamma+3 a_{0}^{2} a_{1} \mu \lambda \gamma=0 \\
& \left(G^{\prime} / G\right): a_{1} \lambda^{2} v^{2}+2 a_{1} \mu \nu^{2}+8 a_{1} \mu \lambda \alpha \nu+a_{1} \lambda^{3} \alpha \nu+16 a_{1} \mu^{2} \beta \\
& +22 a_{1} \mu \lambda^{2} \beta+a_{1} \lambda^{4} \beta+18 a_{0} a_{1}^{2} \mu \lambda \gamma+3 a_{0}^{2} a_{1} \lambda^{2} \gamma+6 a_{0}^{2} a_{1} \mu \gamma \\
& +6 a_{1}^{3} \mu^{2} \gamma=0 \\
& \left(G^{\prime} / G\right)^{2}: 3 a_{1} \lambda \nu^{2}+8 a_{1} \mu \alpha \nu+7 a_{1} \lambda^{2} \alpha \nu+60 a_{1} \mu \lambda \beta+15 a_{1} \lambda^{3} \beta \\
& +15 a_{1}^{3} \mu \lambda \gamma+24 a_{0} a_{1}^{2} \mu \gamma+12 a_{0} a_{1}^{2} \lambda^{2} \gamma+9 a_{0}^{2} a_{1} \lambda \gamma=0, \\
& \left(G^{\prime} / G\right)^{3}: 2 a_{1} v^{2}+12 a_{1} \lambda \alpha \nu+40 a_{1} \mu \beta+50 a_{1} \lambda^{2} \beta+9 a_{1}^{3} \lambda^{2} \gamma \\
& +18 a_{1}^{3} \mu \gamma+30 a_{0} a_{1}^{2} \lambda \gamma+6 a_{0}^{2} a_{1} \gamma=0, \\
& \left(G^{\prime} / G\right)^{4}: 6 a_{1} \alpha \nu+60 a_{1} \lambda \beta+21 a_{1}^{3} \lambda \gamma+18 a_{0} a_{1}^{2} \gamma=0 \text {, } \\
& \left(G^{\prime} / G\right)^{5}: 24 a_{1} \beta+12 a_{1}^{3} \gamma=0 \text {. }
\end{aligned}
$$

Solving this system of algebraic equations, with the aid of Mathematica, one possible set of solution is

$$
\alpha=\frac{3 \lambda \sqrt{\beta}}{\sqrt{2\left(\lambda^{2}-\mu\right)}}, \quad v=-\frac{3 \beta \lambda}{\alpha}, \quad a_{0}=0, \quad a_{1}=\sqrt{\frac{-2 \beta}{\gamma}} .
$$

Substituting these values from (34) and the corresponding solution of ODE (13) into (27) yields three types of traveling wave solutions of equation (26) as follows. 
Case 1: When $\lambda^{2}-4 \mu>0$, we obtain the hyperbolic function solution

$$
U_{1}(x, t)=\sqrt{\frac{-2 \beta}{\gamma}}\left[-\frac{\lambda}{2}+\delta_{1}\left(\frac{C_{1} \sinh \left(\delta_{1} z\right)+C_{2} \cosh \left(\delta_{1} z\right)}{C_{1} \cosh \left(\delta_{1} z\right)+C_{2} \sinh \left(\delta_{1} z\right)}\right)\right]
$$

where $z=x-v t, \delta_{1}=\frac{1}{2} \sqrt{\lambda^{2}-4 \mu}$ and $C_{1}$ and $C_{2}$ are arbitrary constants.

Case 2: When $\lambda^{2}-4 \mu<0$, we obtain the trigonometric function solution

$$
U_{2}(x, t)=\sqrt{\frac{-2 \beta}{\gamma}}\left[-\frac{\lambda}{2}+\delta_{2}\left(\frac{-C_{1} \sin \left(\delta_{2} z\right)+C_{2} \cos \left(\delta_{2} z\right)}{C_{1} \cos \left(\delta_{2} z\right)+C_{2} \sin \left(\delta_{2} z\right)}\right)\right],
$$

where $z=x-v t, \delta_{2}=\frac{1}{2} \sqrt{4 \mu-\lambda^{2}}$ and $C_{1}$ and $C_{2}$ are arbitrary constants.

Case 3: When $\lambda^{2}-4 \mu=0$, we obtain the rational function solution

$$
U_{3}(x, t)=\sqrt{\frac{-2 \beta}{\gamma}}\left(-\frac{\lambda}{2}+\frac{C_{2}}{C_{1}+C_{2} z}\right)
$$

where $z=x-v t$, and $C_{1}$ and $C_{2}$ are arbitrary constants.

\section{Conclusion}

In this paper, we studied two nonlinear partial differential equations that appear in a variety of scientific fields. These are the modified Kortweg de Vries equation and the higherorder modified Boussinesq equation with damping term. We used the $\left(G^{\prime} / G\right)$-expansion method to obtain exact solutions of these two evolution equations. By using this method, we have successfully obtained traveling wave solutions expressed in the form of a hyperbolic function, a trigonometric function and a rational function. This work also highlighted the power of the $\left(G^{\prime} / G\right)$-expansion method for the determination of exact solutions of nonlinear evolution equations.

\section{Competing interests}

The authors declare that they have no competing interests.

\section{Authors' contributions}

DMM and CMK worked together in the derivation of the mathematical results. Both authors read and approved the final manuscript.

\section{Acknowledgements}

DMM and CMK would like to thank the organizing Committee of the International Conference on the Theory, Methods and Application of Nonlinear Equations for their kind hospitality during the conference. DMM would also like to thank the Faculty Research Committee of FAST, North-West University for their financial support.

Received: 5 March 2013 Accepted: 24 May 2013 Published: 12 June 2013

\section{References}

1. Wazwaz, A-M: A modified KdV-type equation that admits a variety of travelling wave solutions: kinks, solitons, peakons and cuspons. Phys. Scr. 86, Article ID 045501 (2012)

2. Yan, Z-Y, Xie, F-D, Zhang, H-Q: Symmetry reductions, integrability and solitary wave solutions to higher-order modified Boussinesq equations with damping term. Commun. Theor. Phys. 36, 1-6 (2001)

3. Wang, M, Zhou, Y, Li, Z: Application of a homogeneous balance method to exact solutions of nonlinear equations in mathematical physics. Phys. Lett. A 216, 67-75 (1996)

4. $\mathrm{Hu}, \mathrm{JL}$ : Explicit solutions to three nonlinear physical models. Phys. Lett. A 287, 81-89 (2001)

5. $\mathrm{Hu}, \mathrm{JL}$ : A new method for finding exact traveling wave solutions to nonlinear partial differential equations. Phys. Lett. A 286, 175-179 (2001)

6. Lou, SY, Lu, JZ: Special solutions from variable separation approach: Davey-Stewartson equation. J. Phys. A, Math. Gen 29, 4209-4215 (1996) 
7. Ablowitz, MJ, Clarkson, PA: Soliton, Nonlinear Evolution Equations and Inverse Scattering. Cambridge University Press, Cambridge (1991)

8. Gu, CH: Soliton Theory and Its Application. Zhejiang Science and Technology Press, Zhejiang (1990)

9. Matveev, VB, Salle, MA: Darboux Transformation and Soliton. Springer, Berlin (1991)

10. Hirota, R: The Direct Method in Soliton Theory. Cambridge University Press, Cambridge (2004)

11. Yan, ZY: A reduction $m K d V$ method with symbolic computation to construct new doubly-periodic solutions for nonlinear wave equations. Int. J. Mod. Phys. C 14, 661-672 (2003)

12. Yan, ZY: The new tri-function method to multiple exact solutions of nonlinear wave equations. Phys. Scr. 78, Article ID 035001 (2008)

13. Yan, ZY: Periodic, solitary and rational wave solutions of the $3 D$ extended quantum Zakharov-Kuznetsov equation in dense quantum plasmas. Phys. Lett. A 373, 2432-2437 (2009)

14. Lu, DC, Hong, BJ: New exact solutions for the $(2+1)$-dimensional generalized Broer-Kaup system. Appl. Math. Comput. 199, 572-580 (2008)

15. Wazwaz, M: The tanh and sine-cosine method for compact and noncompact solutions of nonlinear Klein-Gordon equation. Appl. Math. Comput. 167, 1179-1195 (2005)

16. Lu, DC: Jacobi elliptic functions solutions for two variant Boussinesq equations. Chaos Solitons Fractals 24, 1373-1385 (2005)

17. Yan, ZY: Abundant families of Jacobi elliptic functions of the $(2+1)$-dimensional integrable Davey-Stewartson-type equation via a new method. Chaos Solitons Fractals 18, 299-309 (2003)

18. Wang, M, Li, X: Extended F-expansion and periodic wave solutions for the generalized Zakharov equations. Phys. Lett. A 343, 48-54 (2005)

19. He, JH, Wu, XH: Exp-function method for nonlinear wave equations. Chaos Solitons Fractals 30, 700-708 (2006)

20. Wang, M, Li, X, Zhang, J: The ( $\left.G^{\prime} / G\right)$-expansion method and travelling wave solutions of nonlinear evolution equations in mathematical physics. Phys. Lett. A 372, 417-423 (2008)

21. Bekir, A, Aksoy, E: The exact solutions of shallow water wave equation by using the $\left(G^{\prime} / G\right)$-expansion method. Waves Random Complex Media 22(3), 317-331 (2012)

22. Li, L-X, Wang, M-L: The $\left(G^{\prime} / G\right)$-expansion method and travelling wave solutions for a higher-order nonlinear Schrodinger equation. Appl. Math. Comput. 208, 440-445 (2009)

doi:10.1186/1687-1847-2013-166

Cite this article as: Mothibi and Khalique: On the exact solutions of a modified Kortweg de Vries type equation and higher-order modified Boussinesq equation with damping term. Advances in Difference Equations 2013 2013:166.

\section{Submit your manuscript to a SpringerOpen ${ }^{\odot}$ journal and benefit from:}

- Convenient online submission

Rigorous peer review

- Immediate publication on acceptance

- Open access: articles freely available online

- High visibility within the field

- Retaining the copyright to your article 